\title{
Predicting Value of Growth Differentiation Factor 15 and Its Correlations With Atrial Fibrillation
}

\author{
Jing Zhou, BS ${ }^{1}$, Zhefeng Kang, BS ${ }^{2}$, Lulu Liu, $\mathrm{MS}^{2}$, Yingqiang Guo, $\mathrm{MD}^{2}$, Sai Chen, $\mathrm{MD}^{2}$ \\ ${ }^{1}$ Department of Emergency, West China Hospital of Sichuan University, Chengdu, Sichuan, China; ${ }^{2}$ Department of Cardiovascular \\ Surgery, West China Hospital of Sichuan University, Chengdu, Sichuan, China
}

\section{ABSTRACT}

Aims: Despite several clinical risk factors for atrial fibrillation (AF), some newly identified biomarkers may also potentially serve as risk factors for AF. However, none of these factors so far have been adopted in clinical practice. Recently, a number of studies with an attempt to identify the role of growth differentiation factor 15 (GDF-15) in AF have obtained ambiguous results. We try to identify the predicting role of GDF-15 in AF and AF-related complications with meta-analysis or systematic analysis.

Methods and results: We enrolled 10 studies, looking at the predicting role of GDF-15 in non-valvular AF using meta-analysis, summarized its role in AF-related major complications, and discussed whether it was dependable to forecast postoperative AF. It turned out that GDF-15 is an independent factor to predict occurrence of AF, while it remains obscure to directly demonstrate its relationship with postoperative AF. For AF patients on anti-platelet treatment, GDF-15 plays a critical role in predicting major bleeding, cardiovascular death and overall death, and improves the current predicting model.

Conclusions: Circulating GDF-15 greatly associates with $\mathrm{AF}$ and $\mathrm{AF}-$ related complications. It should be applied clinically.

\section{INTRODUCTION}

Atrial fibrillation (AF) is the most common arrhythmia worldwide, and the population affected is projected to double over the next two decades [Vergara 2014]. It arises from different etiologies: many cardiovascular diseases, such as coronary heart disease, rheumatic heart disease, cardiomyopathy, hypertension, pericardial disease, congenital heart disease and other cardiovascular diseases may result in this same clinical manifestation and seriously affect human health [Shiran 2009; Hirsh 2015; Andrade 2014]. AF is directly associated with numerous cerebrovascular and cardiovascular events, which causes a high morbidity of complications and mortality. However, prediction of $\mathrm{AF}$ has

Received December 12, 2018; accepted September 8, 2019.

Correspondence: Sai Chen, MD, Department of Cardiovascular Surgery, West China Hospital of Sichuan University (e-mail: 61751557@qq.com). long been a bottleneck for scientific researchers, due to its complex etiology and extensive interdependence with other cardiovascular diseases, albeit with atrial fibrosis and electrical remodeling being accepted as the basic manifestations of patients with AF [Adam 2015; Zhang 2015]. These pathological identifications may specify causes or results of AF, but hardly can be used as clinical predictors.

So far, several models (e.g. CSADS2 and CSA2DS2VASC for stroke; HAS-BLED, ORBIT and ABC-bleeding score for bleeding; ABC-death risk score for death) have been established to predict occurrence of AF-related adverse events when patients receive anticoagulation therapy, whereas none has been applied to predicting occurrence of AF. Better predictive efficiency is strongly expected.

Growth differentiation factor-15(GDF-15) is a distantmember of transforming growth factor-beta super family (TGF- $\beta$ ). It is expressed in response to inflammation and stress (e.g. mechanical, ischemic, and oxidative stress, etc.) in a number of cells, such as myocytes and adipocytes [Kempf 2011; Schlittenhardt 2004]. Recent studies found surged expressions of GDF-15 in peripheral blood and myocardial tissue in various cardiovascular diseases including coronary heart disease, ischemia reperfusion injury, and heart failure, etc., some even suggested that GDF-15 may provide prognostic values [Kahli 2014; Fan 2014; Fuernau 2014]. Moreover, several studies attempted to identify whether GDF-15 was associated with $\mathrm{AF}$ or AF-related adverse events. Despite consistent results of the relationship between GDF-15 and AFrelated adverse events summarized from two large-scale multicenter retrospective trials, equivocal conclusions for the association of GDF-15 and AF still exist. Therefore, we aimed to decide whether GDF-15 really assisted to improve current predicting models for $\mathrm{AF}$ and $\mathrm{AF}$-related diseases and summarize current understandings about the relationship between GDF-15 and AF.

Supplemental Data 1. Medline (pubmed), EMBASE, and Cochrane Library search strategy was done with free text search terms. Free text terms search strategy: 1 AND 2 or 3 or 4.

\begin{tabular}{|c|c|c|}
\hline Term 1 & “and" & Term 2 \\
\hline \multirow[t]{3}{*}{1 - atrial fibrillation } & $2-g$ & ion factor-15 \\
\hline & 3- $g r$ & on factor 15 \\
\hline & & \\
\hline
\end{tabular}


Table 1. Flow diagram showing the selection of included studies

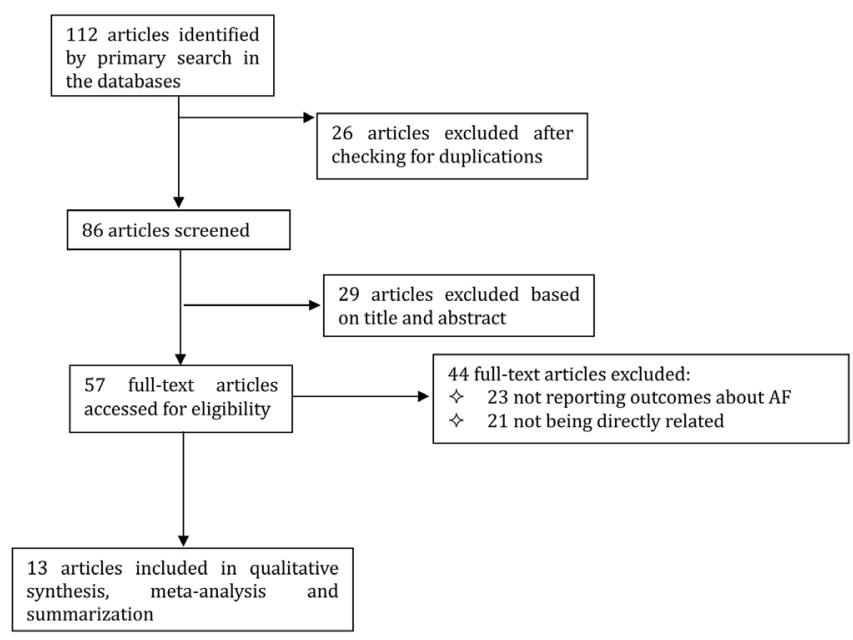

In this study, we aimed to clarify whether GDF-15 serves as an independent factor predicting AF, and its role in improving the risk stratification concerning complications in patients with $\mathrm{AF}$ receiving anticoagulants.

\section{METHODS}

Study inclusion criteria: Three retrospective studies investigating the predicting value of GDF-15 on AF as well as two multiple-center subcohorts, regarding the relationship between GDF-15 and AF-related adverse outcomes, were included in this meta-analysis. These analyses were performed in accordance with the guidelines for Quality of Reporting of Meta-analysis (QUORUM, MOOSE) [Moher 1999]. The remaining one study focused on the relationship of GDF-15 and postoperative AF (POAF), and four studies explored the role of GDF-15 in heart failure-related AF. We included studies that met the following specified criteria: (a) exploration of the predicting value of GDF-15 for AF; (b) the usefulness of GDF-15 in risk stratification concerning complications in patients with AF receiving anticoagulants; (c) relationship of GDF-15 and other cardiovascular diseases that may cause AF.

Search strategy: We searched PubMed, EMBASE, and the Cochrane Database for all published studies that detected GDF-15 and explored any possible relationship with AF up to December 2018, using a predefined keyword list (Supplementary Data S1: search strategy). There were no language restrictions. A reference management software Endnote X7 database was used to organize all titles and abstracts. Potentially relevant abstracts were reviewed after initial abstract identification with subsequent full-text evaluation. References of relevant reports and reviews were screened to identify other eligible studies. When more than one publication from the same patient cohort existed, the study with the most complete dataset was included in the systematic review.
Table 2. Baseline characteristics in the PIVUS (978), USLAM (725) and CBFOC (3217) cohorts

\begin{tabular}{lccc}
\hline & PIVUS & ULSAM & CBFOC \\
\hline Country & Sweden & Sweden & U.S. \\
Population & 978 & 725 & 3217 \\
Follow-up (year) & 10.0 & 7.9 & 10.0 \\
Age & $70.1(0.1)$ & $77.5(0.7)$ & $59(10)$ \\
Sex (\% females) & 51 & 0 & 54 \\
Systolic blood pressure (mm Hg) & $149(22)$ & $151(20)$ & $128(19)$ \\
Current smoking (\%) & 11 & 8.3 & 16 \\
Diabetes mellitus (\%) & 12 & 15 & 11 \\
Prevalent myocardial infarction (\%) & 7 & 13 & 4 \\
Prevalent heart failure (\%) & 4 & 6 & 1 \\
Antihypertensive treatment (\%) & 32 & 41 & 27 \\
\hline
\end{tabular}

Data are means ( \pm standard deviation) or proportions (\%). PIVUS, Prospective Investigation of the Vasculature in Uppsala Seniors; ULSAM, Uppsala Longitudinal Study of Adult Men; CBFOC, Framingham Heart Study Offspring Cohort

Data extraction and quality assessment: We extracted all relevant data, including demographic data, hazard ratio (HR), concentrations of GDF-15 at different points in time, c-statistics with or without GDF-15 for prediction, and information about end-points of interest. Consensus was reached by discussion and recognition of errors in case of disagreements.

Statistical analysis: The main results consisted of pooled risk ratio (HR) with 95\% confidence interval (CI) showing the role of GDF-15 in predicting AF. Statistical analyses were performed by Stata (Version 14.0). Estimated effects were expressed as the relative risk (HR), and the $95 \%$ confidence interval $(95 \% \mathrm{CI})$ were used and summarized by Forest plots. Q-statistics $(P<0.01)$ or $\mathrm{I}^{2}$-statistics $\left(\mathrm{I}^{2}>50 \%\right)$ were performed to test for heterogeneity between included studies [Cleophas 2007]. In the absence of heterogeneity, a standard fixed-effects model (Mantel-Haenszel model) was used; otherwise, the DerSimonian and Laird random-effects model was implemented [DerSimonian 1986]. Publication bias was assessed by visual assessment of funnel plots. Publication bias additionally was examined by Harbord weighted regression statistic with a $P$-value $<0.05$ indicating significant publication bias among included studies. In this case, the Trim-and-Fill method by Duval and Tweedie was used for correction of publication bias [Duval 2000]. Since the studies investigating the relationship between GDF-15 and $\mathrm{AF}$ are limited, no significant heterogeneity or publication bias has been found. The main results of c-statistics for assessment of GDF-15 were summarized in tables and figures. The authors had full access to the data and take responsibility for its integrity. All authors have read and agreed to the manuscript as written. 


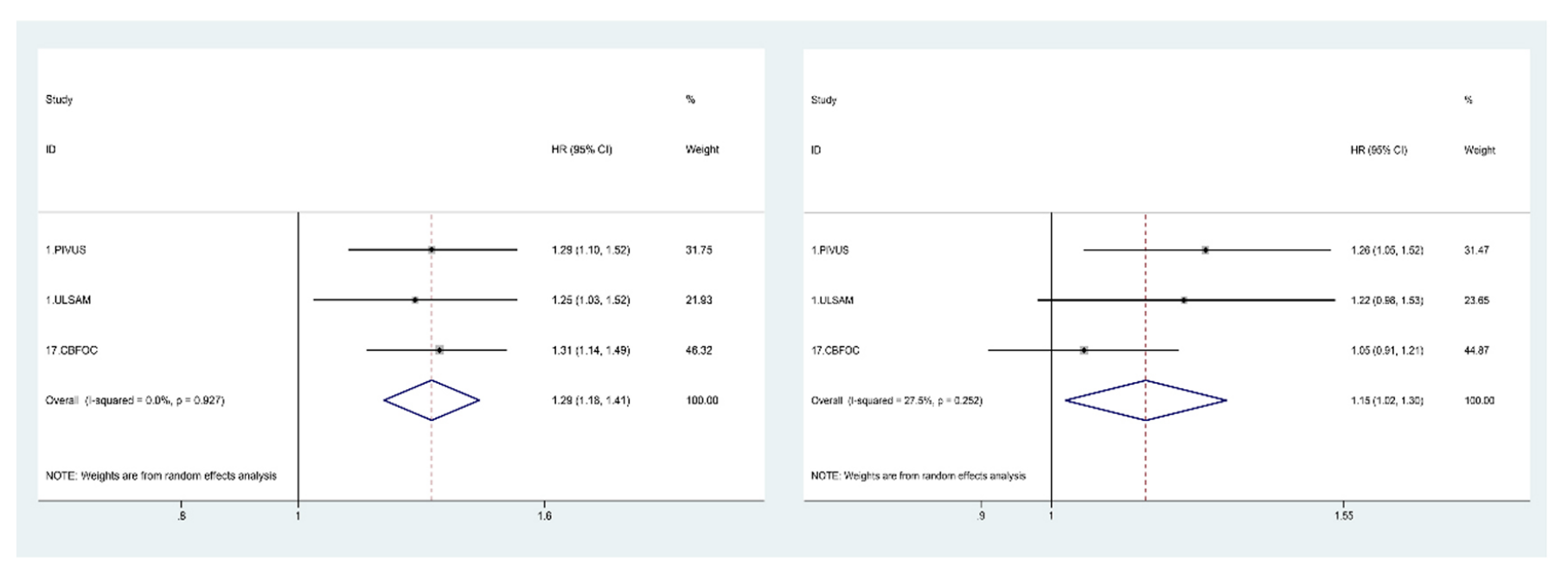

Figure 1. Pooled results on the predicting role of GDF-15 in AF. A) Assessment of predictive value of GDF-15 after adjustment for age. B) Assessment of predictive value of GDF-15 after further adjustment for clinical risk factors and biomarkers.

\section{RESULTS}

References screening: In our database screen, 109 studies were retrieved. These include nine references from Cochrane Library references, 82 references from Embase, and 28 from Pubmed (Medline). Twenty-six references were excluded, due to duplication detected by EndNote software filtration, and the same for another 29 references after reading the title and abstract because they were irrelevant to the current study. Therefore, 54 references were considered for further review. After reading the full texts of the articles, 23 references were excluded, as they did not report outcomes about atrial fibrillation. Another 21 references were excluded because the relationship between GDF-15 and AF was not specified. Consequently, 10 studies were included in the final analysis (Table 1).

GDF-15 serves as an independent factor that predicts AF: The meta-analysis combined individual data extracted from three different community-based cohorts of elderly subjects which included GDF-15 as a potential risk marker for incident AF. In PIVUS (Prospective Investigation of the Vasculature in Uppsala Seniors), 148 participants developed AF, during a median 10-year follow-up in a study sample that consisted of 978 individuals free of AF at baseline at age 70. In ULSAM (Uppsala Longitudinal Study of Adult Men), 123 participants developed $\mathrm{AF}$, during a 7.9-year follow-up in a cohort with a population of 725 subjects free from known AF at baseline at age 77 [Lind 2017]. In CBFOC (Framingham Heart Study Offspring Cohort), 242 incident $\mathrm{AF}$ events occurred during 10 years of follow-up in 3,217 individuals without $\mathrm{AF}$ at baseline at age 59 [Rienstra 2014]. Baseline characteristics were presented in Table 2.

Each study independently investigated the relationship between GDF-15 and AF. Overall, a total of 4,020 patients were included, and $513(12.76 \%)$ patients developed $\mathrm{AF}$ during follow up. Figure 1 shows the combined results of ULSAM, PIVUS and CBFOC, regarding the predictive role of GDF-15 in AF adjusted for age or multiple factors, respectively. (Figure 1) It turned out that GDF-15 significantly was associated with $\mathrm{AF}$ (HR:1.29, 95\% CI:1.28-1.41, $P=.000$, $\left.\mathrm{I}^{2}=0.0 \%\right)$ when adjusted for age. That association remained obvious even adjusted for multiple clinical factors and biomarkers (HR:1.15, 95\% CI:1.02-1.30, $P=.022, \mathrm{I}^{2}=27.5 \%$ ).

GDF-15 with heart failure- or postoperative-related AF: Doulamis et al generally discovered in their proteomic profile study that GDF-15 could be helpful in stratifying risk for AF in patients with cardiovascular disease [Doulamis 2018]. This echoed with previous study that emergence of AF indeed signaled poorer prognosis for patients with heart failure. Four studies gained the same results and identified that in patients with heart failure, GDF-15 were positively related to AF. GDF-15 is similarly elevated in HFpEF (heart failure with preserved ejection fraction) and HFrEF (heart failure with reduced ejection fraction), while it expands its expression when AF occurs [Chan 2016; Izumiya 2014; Montoro-Garcia 2012; Anand 2010]. Thus, monitoring GDF-15 may actively prevent $\mathrm{AF}$ in patients with $\mathrm{HF}$.

So far, only one study attempted to investigate the relationship between GDF-15 and postoperative AF (POAF) [Bouchot 2015]. To our surprise, they were negatively related. The authors proposed that since GDF-15 was a cardioprotective cytokine, low circulating levels of GDF-15 may signal limited ability of self-protection and significant inclination to POAF. However, this result still contradicts current understandings about GDF-15 because younger and healthier individuals generally possess lower circulating levels of GDF-15 [Wollert 2017]. We hypothesized that there were limitations for merely considering concentrations of GDF-15. For many patients, since GDF-15 serves as a cardioprotective cytokine to combat cardiac lesions, higher levels of GDF-15 may suggest more severe cardiovascular injury. While for some other patients, they could possibly suffer from inability to produce enough GDF-15, thus lower concentrations of GDF-15 may forebode vulnerability in self-protection. Since only one study on GDF-15 in the field of POAF was published so far, more research is warranted. 
$\mathrm{HR}(95 \% \mathrm{Cl})$

stroke/systemic embolism, model A PIVUS subcohort

ARISTOTLE subcohort

Subtotal $($ I-squared $=0.0 \%, p=0.663$ )

stroke/systemic embolism, model B

PIVUS subcohort

ARISTOTLE subcohort

Subtotal $($ I-squared $=0.0 \%, p=0.965)$

major bleeding,model $\mathrm{A}$

PIVUS subcohort

ARISTOTLE subcohort

Subtotal (I-squared $=23.9 \%, p=0.252$ )

major bleeding,model B

PIVUS subcohort

ARISTOTLE subcohort

Subtotal $($ I-squared $=0.0 \%, p=0.623$ )

death, model A

PIVUS subcohort

ARISTOTLE subcohort

Subtotal $(\mathrm{I}-$ squared $=67.9 \%, p=0.078)$

death,model B

PIVUS subcohort

ARISTOTLE subcohort

Subtotal $($ I-squared $=3.2 \%, p=0.310)$

cardiovascular death,model A

PIVUS subcohort

ARISTOTLE subcohort

Subtotal (I-squared $=0.0 \%, p=0.792$ )

cardiovascular death,model B

PIVUS subcohort

ARISTOTLE subcohort

Subtotal $($ I-squared $=0.0 \%, p=0.419$ )

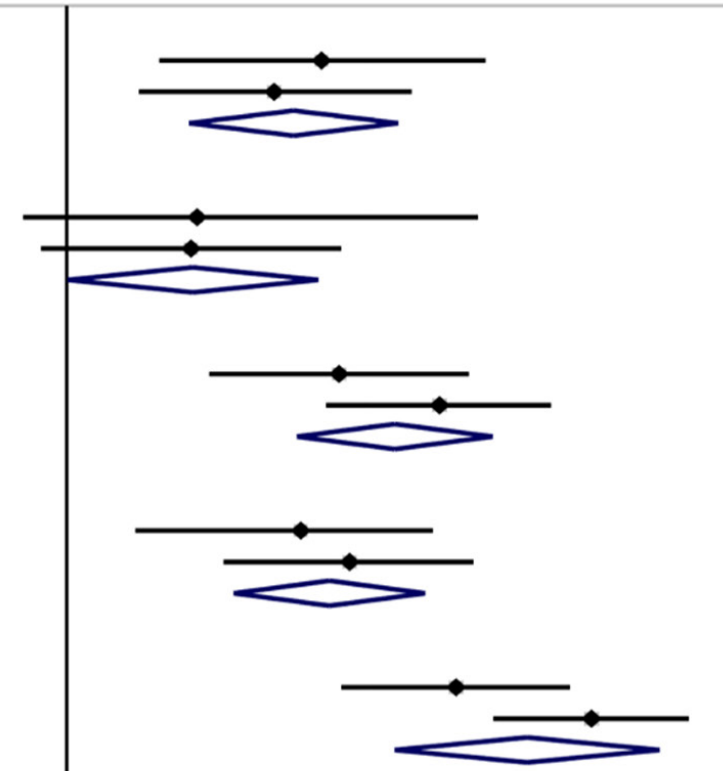

$1.85(1.25,2.75)$

$1.65(1.19,2.30)$

$1.73(1.34,2.23)$

$1.37(0.90,2.70)$

$1.35(0.94,1.94)$

$1.36(1.00,1.83)$

$1.93(1.41,2.64)$

$2.46(1.87,3.22)$

$2.21(1.74,2.80)$

$1.76(1.18,2.42)$

$1.98(1.46,2.67)$

$1.89(1.50,2.38)$

$2.56(1.94,3.37)$

$3.55(2.80,4.49)$

$3.04(2.21,4.19)$

$1.72(1.30,2.29)$

$2.10(1.62,2.73)$

$1.92(1.58,2.33)$

$3.16(2.19,4.54)$

$2.96(2.14,4.08)$

$3.05(2.39,3.88)$

$1.83(1.26,2.66)$

$1.48(1.04,2.11)$

$1.64(1.27,2.12)$

Figure 2. Combined results of the sensitivity analyses of the associations between groups of GDF-15 at baseline and cardiovascular events in multivariable analyses. Model A, medications and clinical characteristics. Model B, medications, clinical characteristics and cardiac biomarkers.

GDF-15 and its correlations with risk stratification concerning complications in patients with $\mathrm{AF}$ receiving anticoagulants - baseline characteristics of RE-LY and ARISTOTLE biomarker subcohorts: The Apixaban for Reduction in Stroke and Other Thromboembolic Events in Atrial Fibrillation (ARISTOTLE) trial enrolled patients with $\mathrm{AF}$ who had more than one CHADS2 risk factor for stroke. All patients were randomized in a 1:1 fashion to doseadjusted warfarin or apixaban $5 \mathrm{mg}$ twice daily. We included the ARISTOTLE biomarker subcohort, consisting of 14,798 patients with measurements of GDF-15 and other biomarkers at randomization [Wallentin 2014]. The Randomized Evaluation of Long-Term Anticoagulation Therapy (RE-LY) trial recruited patients from 967 centers between November 2005 and December 2007 in 44 countries. All patients with AF who had more than one additional risk factor for stroke were randomized to adjusted-dose warfarin or fixed doses of dabigatran- 110 or $150 \mathrm{mg}$ twice daily (target international normalized ratio 2.03.0) [Hijazi 2017]. We included the RE-LY biomarker subcohort based on 8,474 patients with GDF-15 and other biomarker measurements available at randomization in this analysis. The characteristics of the two subcohorts were summarized in Table 3. 
Table 3. Demographics and baseline characteristics of RE-LY (external validation) and ARISTOTLE (internal derivation) subcohorts

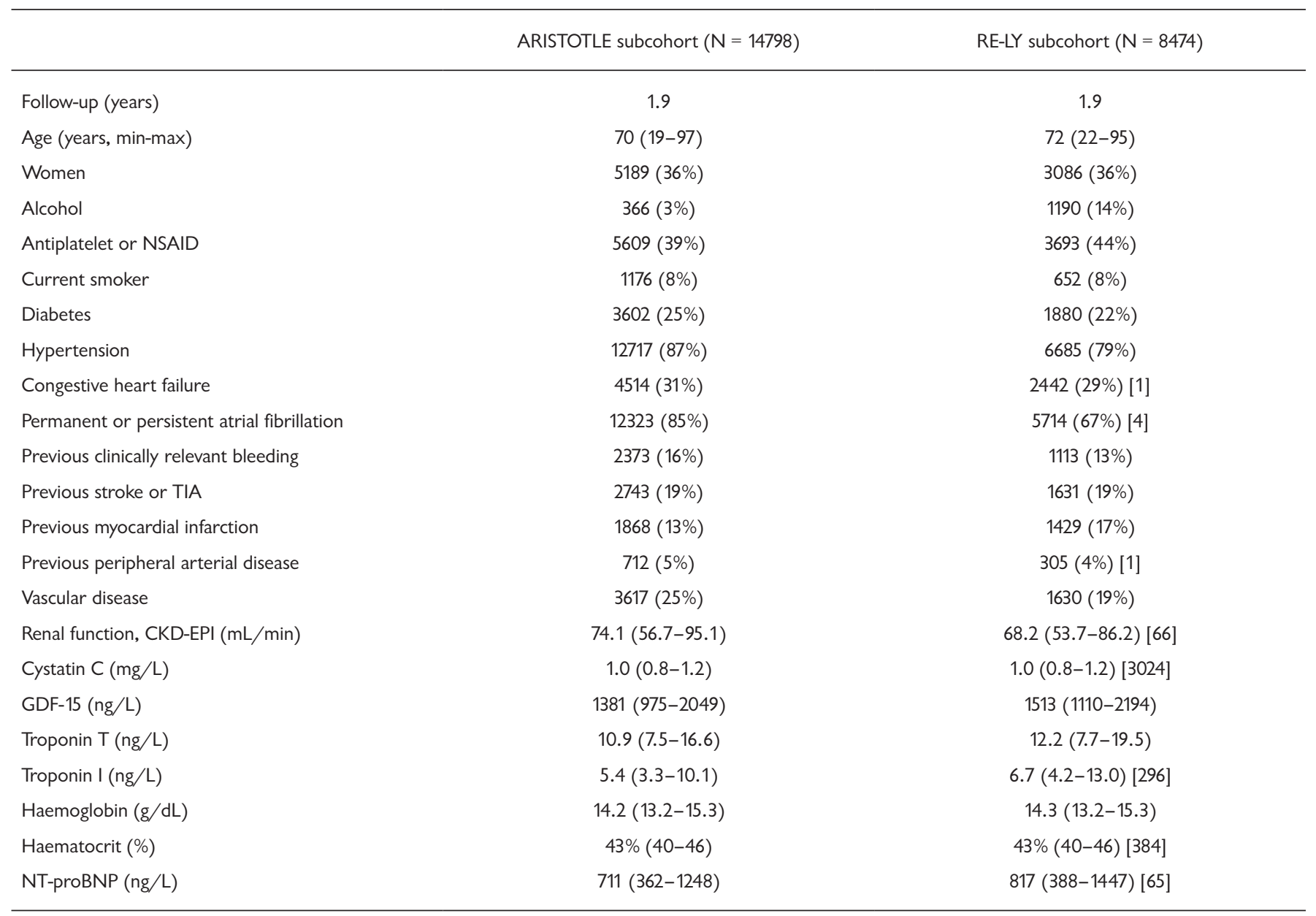

Data are $\mathrm{n}(\%)$ or median (IQR). Numbers within square brackets indicate number of missing values. TIA=transient ischaemic attack. CKD-EPI=Chronic Kidney Disease Epidemiology Collaboration equation. GDF-15=growth differentiation factor 15 . NT-proBNP=N-terminal pro B type natriuretic peptide

GDF-15 and AF-related stroke: The aim of anticoagulation therapy in atrial fibrillation is to obtain a balance between the decrease in ischemic stroke and augment in major bleeding. The RE-LY and ARISTOTLE subcohorts have systematically investigated the risks of anticoagulation therapy for patients with AF. Both studies have drawn similar conclusions and included GDF-15 as risk prediction of stroke. In the RE-LY subcohort, there were 223 events $(1.34 \% / y)$ of systemic embolism or stroke. The baseline GDF-15 concentration strongly was associated with stroke when adjusted for multivariable model A (medications and clinical risk factors). While in further adjustment for model B (cardiac biomarkers troponin and NT-proBNP), GDF-15 became non-associated (adjusted HR [the highest group vs the lowest group] 1.31 [0.86-2.01], $P=.16)$. Similar results also have been gained in the ARISTOTLE subcohort in which $396(1.40 \% / y)$ experienced systemic embolism or stroke within the median followup of 1.9 years. The association of GDF-15 and AF was significant after adjusted for baseline characteristics (model A). After further adjusted for cardiac biomarkers, the association was no longer significant. The combined results of these two analyses also suggest that GDF-15 may not be an independent factor to predict new onset of AF [Hijazi 2017] (Figure 2). Hu et al drew an interesting conclusion that for patients without anticoagulation therapy, elevated GDF-15 independently indicates a significantly increased risk for left atrium/ left atrium thrombus in nonvalvular atrial fibrillation (NVAF) patients [Hu 2018]. However, whether that directly promotes onset of stroke still remains to be seen.

GDF-15 and AF-related bleeding: In the RE-LY subcohort, GDF-15 was not only significant after adjustment for model A, but also proved a predictive value after adjusted for model B for major bleeding. There were $458(2.76 \% / y)$ events of major bleeding during the follow up of 1.9 years. The annualized rate of major bleeding surged from $1.24 \%$ in the lowest group (GDF-15 levels $<1200 \mathrm{ng} / \mathrm{L}$ ) to $4.64 \%$ in the highest group (GDF-15<1800 ng/L). After adjustment for model $\mathrm{B}$, the HR (the highest group vs. the lowest group) was 1.76 ([95\% CI 1.28-2.42], $P=.0003)$. Interestingly enough, GDF-15 itself was able to yield a c-index of 0.67 for 
Table 4. Addition of GDF-15 significantly improves the predictive precision of different models for major bleeding and all-cause mortality.

$$
\text { c- Index }(95 \% \mathrm{Cl})
$$

\begin{tabular}{lcccc} 
Outcomes & Model & Without GDF-15 & With GDF-15 & \\
\hline GDF-15 & & & & \\
All-cause mortality & Only GDF-15 & NA & $0.68(0.65-0.70)$ & NA \\
Major bleeding & Only GDF-15 & NA & $0.67(0.65-0.69)$ & NA \\
$\begin{array}{l}\text { Established risk factors includ- } \\
\text { ing other biomarkers }\end{array}$ & & & & \\
All-cause mortality & Model B & $0.74(0.72-0.77)$ & $0.75(0.73-0.77)$ & \\
Major bleeding & Model B & $0.71(0.69-0.74)$ & $0.72(0.70-0.75)$ & \\
Clinical scores & & & $0.68(0.66-0.70)$ & $<0001$ \\
All-cause mortality & CHA2DS2VASc & $0.59(0.56-0.61)$ & $0.69(0.67-0.72)$ & $<0.71(0.68-0.73)$
\end{tabular}

Data are c-indexes $(95 \% \mathrm{Cl})$ for each score. Descriptions of the scores: CHA2DS2VASc score-assigns one point each for congestive heart failure, hypertension, diabetes mellitus, vascular disease, age 65-74 years, and gender category (female gender), and two points for age $\geq 75$ years and, prior stroke/transient ischaemic attack; ORBIT score is older age ( $\geq 75$ years), reduced haemoglobin/haematocrit/history of anaemia, bleeding history, insufficient kidney function, and treatment with antiplatelet; and HAS-BLED is hypertension, abnormal renal/liver function, stroke, bleeding history or predisposition, labile INR, elderly (>65), Drugs/alcohol concomitantly).

major bleeding, which already outperformed the HAS-BLED score (with a c index of 0.62) and emulated the ORBIT (with a c index of 0.68). The HAS-BLED and ORBIT scores were improved for predicting major bleeding when GDF-15 was added to both models. GDF-15 improved the $\mathrm{c}$ index of HAS-BLED to $0.69(P<.0001)$ and that of ORBIT to 0.71 $(P<.0001)$. In the ARISTOTLE subcohort, $669(2.61 \% / \mathrm{y})$ experienced major bleeding during the median follow-up period. The annualized rate of major bleeding increased from the lowest group to a 3.5-times-higher level in the highest group. After adjustment for model B, the adjusted HR (the highest group vs. the lowest group) was 1.98 ([95\% CI 1.462.67], $P=.0001)$. Addition of GDF-15 improved the $\mathrm{c}$ index of HAS-BLED from 0.633 to $0.677(P<.0001)$. The analysis of continuous GDF-15 showed an improved $\mathrm{c}$ index of 0.682 from $0.664(P<.0001)$ when GDF-15 was added [Hijazi 2017; Hijazi 2016] (Figure 2) (Table 4).

Hijazi et al even developed a brand new score which outperformed HAS-BLED and ORBIT, based on these two subcohorts. They defined the remaining 14,537 patients in ARISTOTLE as the derivation cohort and the 8,468 in RE-LY as the validation cohort (several patients with missing data had been ruled out from the original subcohorts). Both clinical variables and biomarkers (GDF-15, haemoglobin, cTnT-hs, cystatin C, eGFR, NT-proBNP, and haematocrit) were included in the new biomarker-based bleeding score. The strongest predictors were GDF-15, haemoglobin, cTnT-hs, age, and previous bleeding. That was where the acronym ABC-bleeding (age, biomarkers [GDF-15, cTnT-hs, and haemoglobin], clinical history [previous bleeding]) came from. In the derivation cohort, the ABC-bleeding risk score achieved a c-index of 0.68 (95\% CI 0.66-0.70) compared with 0.61 (95\% CI 0.59-0.63) for HAS-BLED and 0.65 (95\% CI $0.62-0.67)$ for ORBIT $(P<.0001$ and $P=.0008$, respectively). The external validation cohort also obtained similar results. The ABC-bleeding score (with a c-index of 0.71 [95\% CI $0.68-0.73]$ ) significantly overmatched the HAS-BLED (0.62 [95\% CI 0.59-0.64], $P<.0001)$ and ORBIT (0.68 [95\% CI 0.65-0.70], $P=.0016$ ) These data were presented in Table 5 [Hijazi 2016] (Table 5).

GDF-15 and AF-related all-cause/cardiovascular death: In the RE-LY subcohort, a total of $586(3.53 \% / y)$ allcause deaths and $381(2.30 \% / y)$ cardiovascular deaths were observed during the follow up of 1.9 years. The adjusted (after adjusted for model B) HR (the highest group vs. the lowest group) was $1.72(1.30-2.29, P=.0004)$ for all-cause mortality and $1.83(1.26-2.66, P=.0019)$ for cardiovascular death, respectively [Hijazi 2017]. In the ARISTOTLE subcohort, 1,061 (3.69\%/y) died, $537(1.88 \% / y)$ died of cardiac diseases. After adjusted for model B, GDF-15 still provided significant independent prognostic information on total mortality. Cardiac mortality also was associated with higher GDF-15 (four to five times higher rates in the highest group than in the lowest group). The adjusted HR (the highest group vs. the lowest group) was 2.10 (95\% CI 1.622.73) for total mortality and 3.55 (95\% CI $2.80-4.49)$ for cardiovascular mortality. Our pooled results also identified GDF-15 as an independent risk predicting factor (Figure 2). The CHA2DS2VASc score provided a c-index of 0.637 alone and surged to 0.707 in addition of GDF-15 $(P<.0001)$ [Hijazi 2017] (Table 4). 
Table 5. C-indexes for the ABC-bleeding score compared with the HAS-BLED and ORBIT scores in the full cohorts and the subcohort without previous bleeding. Data are c-indexes $(95 \% \mathrm{Cl})$ for each score.

\begin{tabular}{lcc}
\hline & Full cohort & No previous bleeding \\
\hline $\begin{array}{l}\text { Derivation cohort } \\
\text { (events/n in group) }\end{array}$ & $662 / 14537$ & $515 / 12164$ \\
ABC - bleeding & $0.68(0.66-0.70)$ & $0.68(0.65-0.70)$ \\
ABC-bleeding (cTnl-hs) & $0.67(0.65-0.69)$ & $0.67(0.64-0.69)$ \\
ABC-bleeding (cystatin C) & $0.67(0.65-0.69)$ & $0.68(0.65-0.70)$ \\
ABC-bleeding (CKD-EPI) & $0.67(0.65-0.69)$ & $0.68(0.65-0.70)$ \\
ORBIT & $0.65(0.62-0.67)$ & $0.65(0.62-0.67)$ \\
HAS-BLED & $0.61(0.59-0.63)$ & $0.60(0.58-0.63)$ \\
Validation cohort & $463 / 8468$ & $331 / 7355$ \\
(events/n in group) & & \\
ABC-bleeding & $0.71(0.68-0.73)$ & $0.68(0.66-0.71)$ \\
ABC-bleeding (cTnl-hs) & $0.71(0.68-0.73)$ & $0.68(0.66-0.71)$ \\
ABC-bleeding (cystatin C) & $0.68(0.64-0.71)$ & $0.65(0.62-0.69)$ \\
ABC-bleeding (CKD-EPI) & $0.69(0.66-0.71)$ & $0.66(0.63-0.69)$ \\
ORBIT & $0.68(0.65-0.70)$ & $0.64(0.61-0.67)$ \\
HAS-BLED & $0.62(0.59-0.64)$ & $0.57(0.55-0.60)$ \\
\hline
\end{tabular}

Descriptions of the scores: $A B C$-bleeding score is age, biomarkers (troponin-hs, haemoglobin, and GDF-15 or renal function), clinical history (previous bleeding); ORBIT score is older age ( $\geq 75$ years), reduced haemoglobin/haematocrit/history of anaemia, bleeding history, insufficient kidney function, and treatment with antiplatelet; and HAS-BLED is hypertension, abnormal renal/liver function, stroke, bleeding history or predisposition, labile INR, elderly (>65), drugs/alcohol concomitantly). CKD-EPI=chronic kidney disease epidemiology collaboration equation

Hijazi et al also developed an ABC (age, biomarkers, clinical history) death risk score based on these two subcohorts, with GDF-15 being one of the most important predictors. Again, the new score dramatically outperformed the current models for death prediction (the model consisting only of clinical variables and the CHA2DS2-VASc score). In the derivation subcohort (ARISTOTLE), the new score yielded a c-index of 0.74 (95\% CI $0.73-0.76)$ for all-cause mortality and 0.76 (95\% CI 0.74-0.78) for cardiovascular mortality, respectively. In comparison, the prediction model, including only clinical variables, yielded a c-index of $0.68(95 \% \mathrm{CI}$ $0.66-0.70)$ for total death and 0.70 (95\% CI $0.68-0.73)$ for cardiovascular death. The CHA2DS2-VASc score, commonly used for stroke prediction, yielded a c-index of 0.59 (95\% CI $0.57-0.61)$ for all-cause mortality and 0.58 (95\% CI $0.56-0.61)$ for cardiovascular mortality. The validation subcohort also coincided with the results in the derivation cohorts. These statistics were presented in Table 6 [Hijazi 2018].
Table 6. C-indexes for cardiovascular and all-cause mortality according to the $A B C$-death score (including alternative biomarkers) compared with the CHA2DS2VASc in the full cohorts

\begin{tabular}{lcc}
\hline & All-cause mortality & $\begin{array}{c}\text { Cardiovascular } \\
\text { mortality }\end{array}$ \\
\hline Derivation cohort, Events/N & $1047 / 14611$ & $532 / 14611$ \\
ABC-death (cTnT) & $0.74(0.73-0.76)$ & $0.76(0.74-0.78)$ \\
ABC-death (cTnl) & $0.75(0.73-0.76)$ & $0.74(0.73-0.76)$ \\
All clinical information & $0.68(0.66-0.70)$ & $0.70(0.68-0.73)$ \\
CHA ${ }_{2}$ DS 2 VASc & $0.59(0.57-0.61)$ & $0.58(0.56-0.61)$ \\
Validation cohort, Events/N & $594 / 8548$ & $385 / 8548$ \\
ABC-death (cTnT) & $0.74(0.72-0.76)$ & $0.77(0.74-0.79)$ \\
ABC-death (cTnl) & $0.73(0.71-0.75)$ & $0.75(0.73-0.78)$ \\
All clinical information & $0.67(0.65-0.69)$ & $0.68(0.66-0.71)$ \\
CHA $_{2}$ DS $_{2}$ VASc & $0.58(0.56-0.61)$ & $0.59(0.56-0.62)$ \\
\hline
\end{tabular}

All clinical information-a model solely consisting of clinical variables (age, gender, smoking, alcohol, prior stroke/TIA, diabetes, hypertension, heart failure, prior myocardial infarction, peripheral arterial disease, vascular disease, AF-type, and prior bleeding). CHA2DS2-VASc-assigns one point each for congestive heart failure, hypertension, diabetes mellitus, vascular disease, age 65-74 years, and gender category (female gender), and two points for age >_75 years and, prior stroke/transient ischaemic attack). ABC-death, age, biomarkers (cardiac troponin, NT-proBNP, and GDF-15), clinical history of heart failure).

\section{DISCUSSION}

Atrial fibrillation (AF) is the most common arrhythmia and the major cause of embolic stroke worldwide [McManus 2012]. The overall morbidity of $\mathrm{AF}$ is $0.9 \%$, and it greatly increases risk of stroke and even death [Hijazi 2017].

GDF-15 is a member of the transforming growth factor beta (TGF- $\beta$ ) superfamily, and its overexpression could be detected in vascular and myocardial cells upon inflammation and oxidative stress. GDF-15 has been shown to reduce inflammatory responses, which inhibit activation of macrophages, cell apoptosis, cardiac remodeling, and cardiac hypertrophy [Rienstra 2014]. Quite a few studies propose GDF-15 as an independent predictor for adverse complications in patients with acute pulmonary embolism, acute coronary syndrome, and heart failure [Wallentin 2014]. Although Zhou et al confirmed that expression of GDF-15 surged in patients with AF, whether it could be used to predict AF has not extensively been studied [Zhou 2015]. Several studies have agreed that the association between incident AF and GDF-15 was statistically significant in age-adjusted and sex-adjusted models. But ambiguous and even contradictory results were obtained after further adjustment for $\mathrm{CV}$ risk factors [Lind 2017; Rienstra 2014]. In our pooled results, we confirmed that GDF-15 was an independent risk prediction factor for incident AF, which may provide novel insights for prevention of AF. 
GDF-15 is elevated in both HFrEF and HFrEF at a similar level. Elevated GDF-15 and further elevations in the follow-up period identify patients at incremental risks of AF, $\mathrm{HF}$ rehospitalization in both HFrEF and HFpEF or even death, which provides increased prognostic information beyond that offered by biomarkers and standard clinical risk factors [Chan 2016; Izumiya 2014; Montoro-Garcia 2012; Anand 2010]. Therefore, monitoring GDF-15 in patients with $\mathrm{HF}$ may effectively delay the disease progression.

The result regarding the relationship between GDF-15 and POAF needs contemplating. So far, circulating levels of GDF-15 have been suggested to reflect conditions of chronic and acute cellular stress associated with aging and cardiac diseases [Kahli 2014; Wollert 2017]. But the current knowledge about the GDF-15 receptor and the potentially involved signaling pathway have not been fully understood. The authors proposed that the negative association between GDF-15 and POAF was because GDF-15 was a cardioprotective cytokine, low circulating levels of GDF-15 may signal limited ability of self-protection and significant inclination to POAF. We hypothesized that there were limitations for merely considering concentrations of GDF-15. How GDF-15 is produced, and how it targets on GDF-15 receptors as well as the downstream signaling needs elucidating. More studies on POAF are warranted.

We also combined the results from the RE-LY and ARISTOTLE subcohorts with biomarkers detected in anticoagulated patients with AF and showed that GDF-15 was consistently and independently associated with risk of major bleeding, cardiovascular mortality and all-cause mortality, but not with stroke or systemic embolic events. Hijazi et al also demonstrated that GDF-15 was the strongest factor associated with major bleeding and death (all-cause and cardiovascular) out of the individual variables, including comorbidities, demographic characteristics, and other biomarkers. If added to HAS-BLED, ORBIT and multiple clinical factors and biomarkers, GDF-15 also could improve the discrimination of major bleeding and death. Thus, GDF-15 seems useful to improve the risk stratification for major bleeding and death. Based on all these new findings, the ABC-bleeding/death risk score (age, biomarkers, and clinical history of bleeding), a novel biomarker-based risk score for major bleeding or death, was developed recently and validated externally in patients with $\mathrm{AF}$ under oral anticoagulation therapy. It consists of the age, biomarkers (cardiac troponin, hemoglobin, and GDF15) as well as clinical history. ABC-bleeding/death score has been shown to significantly outperform the currently used models (HAS-BLED, ORBIT, CSADS2, CSA2DS2VASC, etc.) [Hijazi 2016; Hijazi 2018].

\section{CONCLUSION}

GDF-15 emerges as a new biomarker for risk stratification for cardiac diseases. However, whether it could be used to predict new onset of AF draws ambiguous conclusions. Our analysis first confirmed the possible positive relationship between GDF-15 and AF. We then summarized all current findings of GDF-15 and AF, and further combined results about association between GDF-15 and AF-related complications. Although nearly all current research about GDF-15 on AF have been included in our study, whether it is associated with POAF is still under debate. Further research is warranted.

\section{REFERENCES}

Adam O, Zimmer C, Hanke N, Hartmann RW, Klemmer B, Bohm M, and Laufs U. 2015. Inhibition of aldosterone synthase (CYP11B2) by torasemide prevents atrial fibrosis and atrial fibrillation in mice. Journal of molecular and cellular cardiology. 85(140-50.

Anand IS, Kempf T, Rector TS, Tapken H, Allhoff T, Jantzen F, Kuskowski M, Cohn JN, Drexler H, and Wollert KC. 2010. Serial measurement of growth-differentiation factor-15 in heart failure: relation to disease severity and prognosis in the Valsartan Heart Failure Trial. Circulation. 122(14):1387-95.

Andrade J, Khairy P, Dobrev D, and Nattel S. 2014. The clinical profile and pathophysiology of atrial fibrillation: relationships among clinical features, epidemiology, and mechanisms. Circulation research. 114(9):1453-68

Bouchot O, Guenancia C, Kahli A, Pujos C, Malapert G, Vergely C, and Laurent G. 2015. Low Circulating Levels of Growth Differentiation Factor-15 Before Coronary Artery Bypass Surgery May Predict Postoperative Atrial Fibrillation. Journal of cardiothoracic and vascular anesthesia. 29(5):1131-9.

Chan MM, Santhanakrishnan R, Chong JP, Chen Z, Tai BC, Liew OW, $\mathrm{Ng}$ TP, Ling LH, Sim D, Leong KT, et al. 2016. Growth differentiation factor 15 in heart failure with preserved vs. reduced ejection fraction. European journal of heart failure. 18(1):81-8.

Cleophas TJ, and Zwinderman AH. 2007. Meta-analysis. Circulation. 115(22):2870-5.

DerSimonian R, and Laird N. 1986. Meta-analysis in clinical trials. Controlled clinical trials. 7(3):177-88.

Doulamis IP, Samanidis G, Tzani A, Antoranz A, Gkogkos A, Konstantopoulos P, Pliaka V, Minia A, Alexopoulos LG, Perrea DN, et al. 2018. Proteomic profile of patients with atrial fibrillation undergoing cardiac surgery. Interactive cardiovascular and thoracic surgery.

Duval S, and Tweedie R. 2000. Trim and fill: A simple funnel-plot-based method of testing and adjusting for publication bias in meta-analysis. Biometrics. 56(2):455-63.

Fan LY, Jin ZG, Zong M, Wang QZ, Ju Y, Sun LS, Gu YY, Yu P, Zhang DF, and Liu ZM. 2014. Growth differentiation factor 15, ischemia modified albumin and pregnancy-associated plasma protein A in patients with coronary artery disease. Clinical laboratory. 60(6):973-82.

Fuernau G, Poenisch C, Eitel I, de Waha S, Desch S, Schuler G, Adams V, Werdan K, Zeymer U, and Thiele H. 2014. Growth-differentiation factor 15 and osteoprotegerin in acute myocardial infarction complicated by cardiogenic shock: a biomarker substudy of the IABP-SHOCK IItrial. European journal of heart failure. 16(8):880-7.

Hijazi Z, Oldgren J, Andersson U, Connolly SJ, Eikelboom JW, Ezekowitz MD, Reilly PA, Yusuf S, Siegbahn A, and Wallentin L. 2017. Growthdifferentiation factor 15 and risk of major bleeding in atrial fibrillation: Insights from the Randomized Evaluation of Long-Term Anticoagulation Therapy (RE-LY) trial. American heart journal. 190(94-103. 
Hijazi Z, Oldgren J, Lindback J, Alexander JH, Connolly SJ, Eikelboom JW, Ezekowitz MD, Held C, Hylek EM, Lopes RD, et al. 2016. The novel biomarker-based $\mathrm{ABC}$ (age, biomarkers, clinical history)-bleeding risk score for patients with atrial fibrillation: a derivation and validation study. Lancet. 387(10035):2302-11.

Hijazi Z, Oldgren J, Lindback J, Alexander JH, Connolly SJ, Eikelboom JW, Ezekowitz MD, Held C, Hylek EM, Lopes RD, et al. 2018. A biomarker-based risk score to predict death in patients with atrial fibrillation: the $\mathrm{ABC}$ (age, biomarkers, clinical history) death risk score. European heart journal. 39(6):477-85.

Hijazi Z, Oldgren J, Siegbahn A, and Wallentin L. 2017. Application of Biomarkers for Risk Stratification in Patients with Atrial Fibrillation. Clinical chemistry. 63(1):152-64.

Hirsh BJ, Copeland-Halperin RS, and Halperin JL. 2015. Fibrotic atrial cardiomyopathy, atrial fibrillation, and thromboembolism: mechanistic links and clinical inferences. Journal of the American College of Cardiology. 65(20):2239-51.

Hu XF, Zhan R, Xu S, Wang J, Wu J, Liu X, Li Y, and Chen L. 2018. Growth differentiation factor 15 is associated with left atrial/left atrial appendage thrombus in patients with nonvalvular atrial fibrillation. Clinical cardiology. 41(1):34-8.

Izumiya Y, Hanatani S, Kimura Y, Takashio S, Yamamoto E, Kusaka H, Tokitsu T, Rokutanda T, Araki S, Tsujita K, et al. 2014. Growth differentiation factor-15 is a useful prognostic marker in patients with heart failure with preserved ejection fraction. The Canadian journal of cardiology. 30(3):338-44.

Kahli A, Guenancia C, Zeller M, Grosjean S, Stamboul K, Rochette L, Girard C, and Vergely C. 2014. Growth differentiation factor-15 (GDF15) levels are associated with cardiac and renal injury in patients undergoing coronary artery bypass grafting with cardiopulmonary bypass. PloS one. 9(8):e105759.

Kempf T, Zarbock A, Widera C, Butz S, Stadtmann A, Rossaint J, Bolomini-Vittori M, Korf-Klingebiel M, Napp LC, Hansen B, et al. 2011. GDF-15 is an inhibitor of leukocyte integrin activation required for survival after myocardial infarction in mice. Nature medicine. 17(5):581-8.

Lind L, Sundstrom J, Stenemo M, Hagstrom E, and Arnlov J. 2017. Discovery of new biomarkers for atrial fibrillation using a custom-made proteomics chip. Heart. 103(5):377-82.

McManus DD, Rienstra M, and Benjamin EJ. 2012. An update on the prognosis of patients with atrial fibrillation. Circulation. 126(10):e143-6.
Moher D, Cook DJ, Eastwood S, Olkin I, Rennie D, and Stroup DF. 1999. Improving the quality of reports of meta-analyses of randomised controlled trials: the QUOROM statement. Quality of Reporting of Meta-analyses. Lancet. 354(9193):1896-900.

Montoro-Garcia S, Hernandez-Romero D, Jover E, Garcia-Honrubia A, Vilchez JA, Casas T, Martinez P, Climent V, Caballero L, Valdes M, et al. 2012. Growth differentiation factor-15, a novel biomarker related with disease severity in patients with hypertrophic cardiomyopathy. European journal of internal medicine. 23(2):169-74.

Rienstra M, Yin X, Larson MG, Fontes JD, Magnani JW, McManus DD, McCabe EL, Coglianese EE, Amponsah M, Ho JE, et al. 2014. Relation between soluble ST2, growth differentiation factor-15, and high-sensitivity troponin I and incident atrial fibrillation. American heart journal. 167(1):109-15 e2.

Schlittenhardt D, Schober A, Strelau J, Bonaterra GA, Schmiedt W, Unsicker K, Metz J, and Kinscherf R. 2004. Involvement of growth differentiation factor-15/macrophage inhibitory cytokine-1 (GDF-15/ MIC-1) in oxLDL-induced apoptosis of human macrophages in vitro and in arteriosclerotic lesions. Cell and tissue research. 318(2):325-33.

Shiran A, and Sagie A. 2009. Tricuspid regurgitation in mitral valve disease incidence, prognostic implications, mechanism, and management. Journal of the American College of Cardiology. 53(5):401-8.

Vergara P, and Della Bella P. 2014. Management of atrial fibrillation. F1000Prime Rep. 6(22.

Wallentin L, Hijazi Z, Andersson U, Alexander JH, De Caterina R, Hanna M, Horowitz JD, Hylek EM, Lopes RD, Asberg S, et al. 2014. Growth differentiation factor 15, a marker of oxidative stress and inflammation, for risk assessment in patients with atrial fibrillation: insights from the Apixaban for Reduction in Stroke and Other Thromboembolic Events in Atrial Fibrillation (ARISTOTLE) trial. Circulation. 130(21):1847-58.

Wollert KC, Kempf T, and Wallentin L. 2017. Growth Differentiation Factor 15 as a Biomarker in Cardiovascular Disease. Clinical chemistry. 63(1):140-51.

Zhang L, Huang B, Scherlag BJ, Ritchey JW, Embi AA, Hu J, Hou Y, and Po SS. 2015. Structural changes in the progression of atrial fibrillation: potential role of glycogen and fibrosis as perpetuating factors. International journal of clinical and experimental pathology. 8(2):1712-8.

Zhou YM, Li MJ, Zhou YL, Ma LL, and Yi X. 2015. Growth differentiation factor-15 (GDF-15), novel biomarker for assessing atrial fibrosis in patients with atrial fibrillation and rheumatic heart disease. International journal of clinical and experimental medicine. 8(11):21201-7. 